\title{
Multipacket Reception MAC Schemes for the RFID EPC Gen2 Protocol
}

\author{
Danilo De Donno, Luciano Tarricone \\ Innovation Engineering Department \\ University of Salento \\ Via per Monteroni, 73100, Lecce - Italy
}

\author{
Vasileios Lakafosis, Manos M. Tentzeris \\ Department of Electrical and Computer Engineering \\ Georgia Institute of Technology \\ Technology Square Research Building, Atlanta, GA - USA
}

\begin{abstract}
Maximizing the Radio Frequency IDentification (RFID) performance is one of the main challenges for a wide variety of applications, where the overall throughput can be significantly affected by undesired Tag-Tag collision events. The UHF EPC Class-1 Generation-2 (Gen2) protocol only specifies algorithms to avoid the collisions but makes no provision for their resolution. In this paper, performance enhancement of the RFID EPC Gen2 protocol exploiting Tag collision recovery is demonstrated, for the first time, in real time with measurements. Two simple and effective approaches to handle successful Tag acknowledgments of recovered collided packets are proposed and implemented on a software-defined Reader and programmable Tags. The overall throughput per time slot is increased by $\mathbf{7 2} \%$ over the standard Gen2 MAC scheme. The validity of such result is confirmed by the good agreement with simulations reported in the literature.
\end{abstract}

Keywords-RFID; EPC Gen2 procotol; tag collision; multipacket reception; collision recovery; throughput.

\section{INTRODUCTION}

The Radio-Frequency IDentification (RFID) technology is present in an ever-increasing number of applications, mainly due to its cost-effectiveness and ease of use. It comes as no surprise that, in the effort to automatically identify a vast population of RFID Tags as fast as possible the undesired effect of communication collisions becomes a more and more diffused problem. The direct consequences on the overall throughput are potentially so adverse that even partially solving the problem can bring considerable gains in a vast array of business sectors, such as airports, warehouses and factories [1-3].

The widely known RFID EPC Class-1 Generation-2 ("Gen2" for short hereafter) protocol [4], does not solve the problem. This standard adopts the Framed Slotted Aloha (FSA) [5] and the binary tree splitting algorithms to avoid collisions on the Medium Access Control (MAC) layer. In case of multiple Tags answering simultaneously, a Gen2 compliant RFID Reader (or interrogator) possesses no capabilities of extracting useful information contained within the collided RFID signals; rather, it discards this data, renders the communication with the Tags unsuccessful and just requires a retransmission of the Tag packets, incurring a waste of throughput.
Multipacket reception, as a result of the implementation of collision recovery techniques, has been extensively investigated in the literature [6-13] yielding highly reliable results. The common aspect of these works is the exploitation of diversity-combining techniques for blind source separation, i.e. the separation of independent sources from a mixed signal without having knowledge of the mixing process. The most classical diversity method has been the use of multiple receive antennas [6-9]. Under the single-receive-antenna detection scheme category, Zero Forcing (ZF) and Successive Interference Cancellation (SIC) are used in [10] while Maximum Likelihood (ML) sequence decoders are considered in [11-13]. Although the majority of these works does point out the benefits achievable by implementing multipacket reception in real-world scenarios, only few attempt to quantify the total throughput improvement via simulation. To the best of our knowledge, no work has implemented a real setup with Reader and Tags to leverage the coupling of the collision recovery techniques with minor modifications to the Gen2 protocol to realistically improve the RFID communication performance.

In this paper, throughput improvement with collision recovery is analyzed, for the first time, in real time with actual measurements exploiting the functionality provided by a software-defined RFID Reader and programmable Tags. Essentially, the RFID performance benefits result from very simple, yet effective changes that we propose in the way successful packet acknowledgments from recovered collided packets are handled. This paper demonstrates, for the first time, with a real RFID Gen2 setup the performance boost in the protocol communication that can be drawn by easily and cheaply integrating existing technical contributions to real RFID systems. Our experimental results agree very well with simulations reported in the literature, thus confirming the validity of the proposed approach.

The paper is organized as follows: Section II briefly recalls basics of the Gen2 protocol along with the proposed modifications to exploit Tag collision recovery for multi-Tag acknowledgment; in Section III, some details about the adopted equipment are given while in Section IV the implemented protocol changes are tested with both real-time measurements and simulations. The relevant conclusions are finally drawn in Section V. 


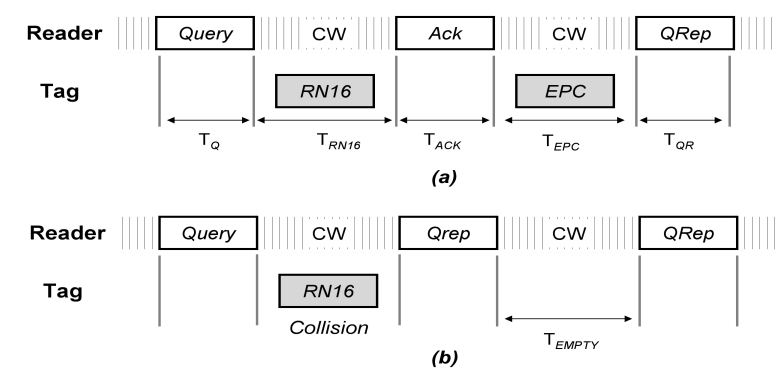

Figure 1. Gen2 protocol in case of single-Tag reply (a) and Tag collision (b).

\section{GEN2 OVERVIEW AND PROPROSED APPROACH}

EPC Gen2 is, nowadays, the most widely adopted passive RFID standard. A Gen2 Reader starts the interrogation procedure by broadcasting a Query command to the Tags. This Query packet not only configures the uplink (from Tag to Reader) communication parameters, such as the encoding scheme (FM0, Miller-2, Miller-4 or Miller-8) and the frequency of the Amplitude Shift Keying (ASK) modulation, but also announces the frame size $K$ (slot $k \in[1, \ldots, K]$ ) of the FSA MAC scheme. A Tag in the interrogation zone of the Reader randomly selects one of these following slots and responds with a random 16-bit number, hereafter referred to as $R N 16$. Upon successful reception, the Reader will echo the $R N 16$ in the following $A C K$ message. If the Tag successfully receives the $A C K$ with the correct (exactly same) RN16 number, it will finally backscatter its 96-bits ID in the EPC message. After all Tags have been read, the Reader will power down. We refer to an individual frame as an Inventory Round, and the series of Inventory Rounds between power-down periods as an Inventory Cycle. Figure 1a shows an example of successful Reader-Tag handshake in the first slot of an Inventory Round. When Tags reply in the same time slot, a collision occurs. In this case, the Reader will not $A C K$ the Tags during the current round (ref. Fig. 1b). However, these Tags will be active in the next round, where they will choose a new random slot. For the rest of the paper, we will refer to Mode 0 as the conventional vanilla Gen2 MAC protocol just described.

Although a typical RFID Reader may only communicate with at most one Tag at a time based on current single-Tag detection techniques, it has been demonstrated that multipacket reception is practically feasible with various novel adhoc algorithms [6-13]. Hence, it is realistic to assume that on the physical layer the Reader can separate and decode collided Tag signals. The question, however, is how can the Reader use the information extracted from the waveform separation. We refer to the simple case where two Tags transmit during the same slot. Note that in such case the collision recovery techniques reported in the literature achieve excellent performance with, for instance, a probability of $85 \%$ to decode both Tag replies reported in [13]. A first possibility for the Reader is to acknowledge only one Tag and discard the other.

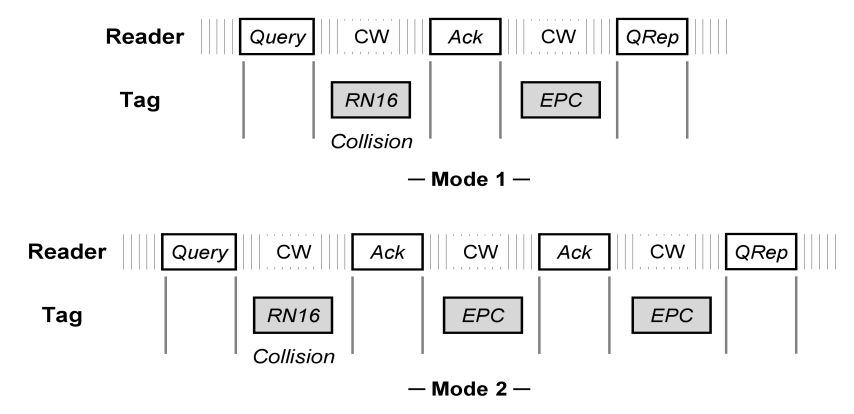

Figure 2. Proposed schemes for the EPC Gen2 MAC protocol in case of twoTag collision recovery.

We refer to this case as Mode 1. Alternatively, the Reader can acknowledge both Tags by sending two consecutive $A C K \mathrm{~s}$ (Mode 2). Figure 2 illustrates the Reader-Tags communication for the two modes. It should be clarified that scenarios involving collisions of more than two Tags are not examined in this work because of the lack of reliable and technically feasible collision-recovery techniques that are directly portable to commercial RFID Readers and can operate in real time. Moreover, two-Tag collisions are the most probable events in real scenarios - three times more probable than collisions involving more than two Tags according to the binomial probability for a population of 100 Tags and 100 allocated time slots. In the rest of the paper, we will use the term "collision" to indicate always two-Tag collision events.

In summary, the two-Tag collision recovery capability allows us to introduce and implement simple (corresponding to only a few lines of code) modifications, namely the aforementioned Mode 1 and Mode 2, to the Gen2 standard to acknowledge collided Tags. The experimental setup used to achieve this goal is introduced in next section.

\section{EXPERIMENTAL SETUP}

We rely on the Intel Wireless Identification and Sensing Platform (WISP) [14] Tags, and on a Software Defined Radio (SDR) RFID Reader [15], [16] as flexible and programmable platforms to implement the proposed modifications to the Gen2 MAC protocol.

The WISP is a passive and programmable RFID Tag developed by Intel Research Seattle. WISP can be powered and read by off-the-shelf UHF RFID Readers and has an onboard microcontroller for sensing and computing functions. The latest firmware version (hw41_D41) that comes with the WISPs is not completely compliant with the EPC Gen2 standard, mainly because of power constraints. For example, the handshake mechanism is not supported and the WISP will always reply to an $A C K$ sent by the Reader, whatever the RN16 contained in it really is. Moreover, the slot selection performed is not genuinely random and the WISPs tend to pick always the same slots. Since the Gen2 handshake and random slot selection functionality are key features for this work, we implemented them on the WISP. As for the slot selection, a simple and computationally inexpensive 
pseudorandom number generator based on a Linear Congruential Generator (LCG) was used. Finally, we programmed the two WISPs used in the experiments with two known RN16 numbers. In fact, since the purpose of this work is not to propose a new technique for separating and decoding collided Tags but instead to highlight the benefits of such a collision recovery, the transmitted RN16 numbers will be assumed known at the Reader. This does not cause loss of generality since it is reliable to assume that well-consolidated and effective multipacket reception techniques will be commonly implemented onto commercial Readers.

The freely available SDR Reader by Buettner [16] is considered in this work as the basis to implement the collisionaware Gen2 MAC protocol. To our knowledge, this is the first and only cost-effective tool, which allows for easy introduction of changes to the PHY and MAC layers of EPC Gen2. It is based on the low-cost Universal Software Radio Peripheral (USRP) [17] and the open-source GNU Radio toolkit [18]. Because the signal processing is completely performed on a standard Linux PC, the SDR Reader enables the modification of MAC and PHY functionalities simply by re-writing user-level software. The effectiveness of GNU Radio and the USRP to investigate RFID communications has been also demonstrated in recent publications [19-21].

The original MAC layer of the SDR Reader has been modified and the two Modes described in Section II implemented. For instance, Fig. 3 depicts a captured communication between the SDR Reader and two collided WISP Tags when Mode 2 is adopted. Preliminary experiments revealed that one of the most critical issues for the Reader is to detect a collision event. In other words, for a given time slot the Reader should be able to determine if no Tag has replied (empty slot), one Tag has replied (single-reply slot), or more than one Tag (exactly two Tags in our case) have replied (collision slot).

In case of collision slot, two possible events may occur at the signal-decoding stage of the Reader:

a) Preamble not found. All Tags modulate the same bits at the beginning (preamble). However, each Tag exhibits its own backscatter delay and link-frequency jitter, due, for example, to imperfections in the low-cost on-board local oscillator, thus making preamble recognition difficult at the Reader.

b) Invalid bit sequences. A collision between Tags in the air interface produces invalid bit sequences at the Reader. The SDR Reader implements Tag decoding using a correlator, i.e. to make a hard decision (bit ' 0 ' or ' 1 ') according to a correlation score. If symbols' score is extremely low, the $R N 16$ packet is marked as invalid.

The tweaked SDR Reader denotes a given slot as collision slot when either one of the following two situations arise:

1. the Signal-to-Noise Ratio (SNR) in the slot is above a fixed threshold (this ensures that at least one Tag has selected the slot) and a valid preamble is not found, or

2. the SNR is above the aforementioned threshold, the preamble is found and the bit sequence is invalid.

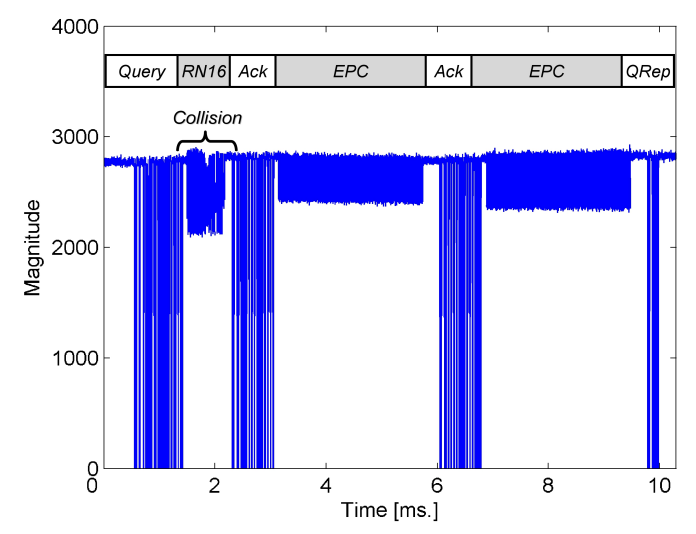

Figure 3. Example of captured Reader-WISPs communication with Mode 2.

In both cases, the chosen SNR threshold is fundamental to minimize the number of situations where a single-reply slot is erroneously marked as collision slot. Based on the analysis of the decoder's performance, we have selected the level of $6 \mathrm{~dB}$ as SNR threshold. Note that the validity of this choice is also confirmed by Buettner's measurements in [15] where more than $95 \%$ of single-reply slots with errors exhibited an SNR of less than $4 \mathrm{~dB}$, i.e. Tag responses with SNR greater than $4 \mathrm{~dB}$ were generally decoded successfully. Therefore, a threshold of $6 \mathrm{~dB}$ ensures that when the preamble is not found or the bit sequence is invalid, a collision slot occurred with high probability.

Once a collision event is detected, we assume that the Reader will implement a multi-packet reception algorithm to separate and decode the Tags' RN16 packets in a real situation, as discussed earlier. Without loss of generality, however, the RN16 values are at this point fixed numbers programmed on the WISPs, so we can consider them known at the Reader. In order to emulate a real scenario, we also added the possibility to choose a collision-recovery probability $p_{C R}$ for the Reader. In other words, the Reader will not always acknowledge Tags, based on the running Mode, when a collision is detected, but only with a certain probability. This mechanism emulates real scenarios where the waveform separation can fail because of noise and multipath fading.

\section{Performance eVAluation}

In order to evaluate the gains achieved by the proposed collision-aware Gen2 protocol, we run a series of experiments in a real Tag-Reader communication scenario. We fix two WISP Tags on a polystirene sheet at a distance of 1 meter from the SDR Reader antennas - note that the USRP-based Reader uses a bistatic configuration, i.e. one antenna is for transmission and one for reception. The Tags and antennas are mounted on easels 1.5 meters above the ground. We instruct the Reader to perform 100 inventory cycles with 5 inventory rounds each. Recall that if a Tag is not successfully singulated by the Reader during a round (for instance, because of a collision event), it will result active in the next round. Conversely, a successfully singulated Tag in a round will be inactive in the next rounds. We repeat the experiments and 


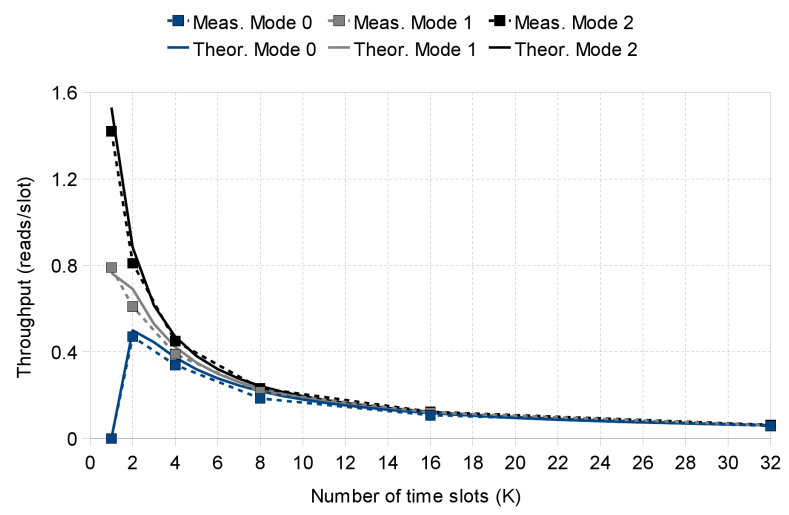

Figure 4. Comparison between theoretical and measured throughput $(\mathrm{N}=2$ Tags).

average out the results provided by the Reader logs for 5 different frequencies in the United States UHF RFID band $(905+i \cdot 5 \mathrm{MHz}$ with $i \in[0,1,2,3,4])$ and for 5 different seeds of the pseudo-random number generator implemented on the WISP Tags for the slot selection.

In order to compare experimental and theoretical results, let us to consider a population of $N$ Tags and inventory rounds consisting of $K$ slots. Tags randomly select one slot $k \in[1, \ldots, K]$ for transmission. As previously discussed, it may happen that certain slots are either not used (empty slots), or used by one Tag (single-reply slot) or used by more than one Tags (collision slots). The probability of $q$ tags transmitting in a given time slot is described by the binomial coefficient:

$$
P(q)_{N, K}=p_{q}=\left(\begin{array}{l}
N \\
q
\end{array}\right)\left(\frac{1}{K}\right)^{q}\left(1-\frac{1}{K}\right)^{N-q}
$$

For Mode 0, a successful Tag transmission occurs if exactly one Tag transmits in a slot (single-reply slot). In that case, the number of Tag reads per slot, i.e. the throughput, is given by:

$$
T_{P}^{\text {Mode } 0}=P(q=1)_{N, K}=N\left(\frac{1}{K}\right)\left(1-\frac{1}{K}\right)^{N-1}
$$

In the outlined scenario, i.e. when two-Tag collisions can be recovered, the theoretical throughput for the two Modes is given by:

$$
\begin{aligned}
& T_{P}^{\text {Mode } 1}=P(q=1)_{N, K}+p_{C D R} \cdot P(q=2)_{N, K} \\
& T_{P}^{\text {Mode } 2}=P(q=1)_{N, K}+p_{C D R} \cdot 2 P(q=2)_{N, K}
\end{aligned}
$$

where $p_{C D R}=p_{C D} \cdot p_{C R}$ is the probability that a collision event can be detected (with probability $p_{C D}$ ) and recovered (with probability $p_{C R}$ ) by the Reader.

The achieved throughput per slot measured in our experiments (recall that the Tag population is $N=2$ ) is reported in Fig. 4 when 0.9 is chosen as probability of collision recovery at the Reader. Figure 4 contains also the theoretical throughput calculated by equations (2), (3) and (4) when $p_{C D}$ and $p_{C R}$ are set to 0.83 and 0.9 respectively. The rationale behind choosing these values is that, regarding the former, a

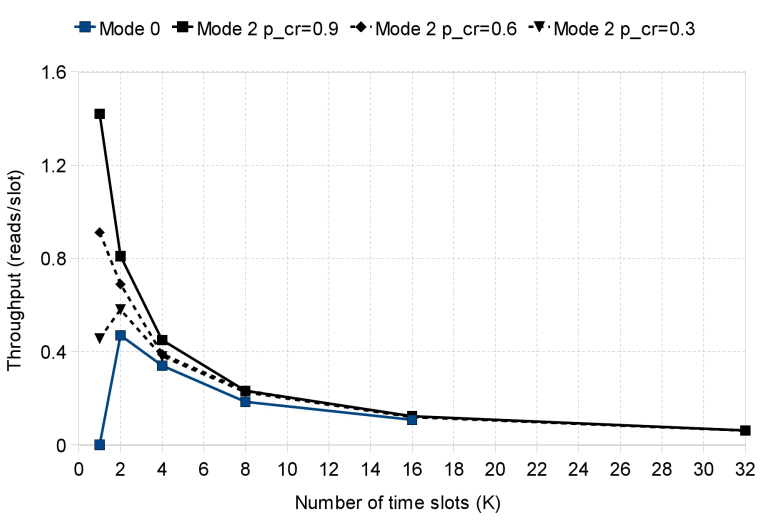

Figure 5. Measured throughput per slot ( $\mathrm{N}=2$ tags) with three different values of collision-recovery probability set for the reader.

collision event is correctly recognized in $83 \%$ of all cases in a set of separate experiments where we forced both Tags to pick the same slot. Regarding the latter, as previously stated, we instructed the reader to implement the collision-aware EPC Gen2 functionalities in $90 \%$ of detected collision events. Imposing in (3) and (4) the empirical values of $p_{C D}$ and $p_{C R}$ is the most suitable way to compare the mathematical formulation to the real experiments.

Differently from Mode 0 where the maximum throughput is achieved when $N=K=2$, the collision-aware Modes achieve best performance when $K=1-$ no read is reported in this case for Mode 0 because Tags are always forced to pick the same slot. When $K=2$ slots are allocated by the Reader, Mode 1 and Mode 2 attain a throughput gain of $30 \%$ and $72 \%$ respectively over Mode 0. This is in quite good agreement with the simulation results reported in [10], where a $60 \%$ increase of the expected throughput for the equivalent of our Mode 1 is achieved - note that the probability of collision detection and recovery are not taken into account in [10].

A parametric analysis for three different values of $p_{C R}$ is also conducted. Figure 5 shows the measured throughput per slot for Mode 0 and Mode 2 when 0.3, 0.6, and 0.9 are set as collision-recovery probabilities for the Reader. It is worth highlighting that even when the Reader can separate a

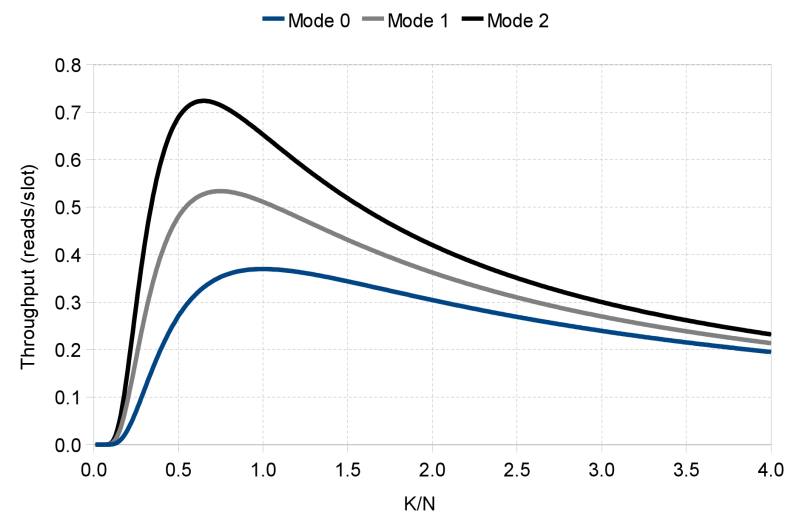

Figure 6. Expected throughput per slot with probabilities of collision detection and recovery set to 0.83 and 0.9 , respectively. 
waveform with merely $30 \%$ of probability, a throughput gain of $23 \%$ is achieved.

We evaluate also the performance improvement, which can be prospectively achieved by the proposed collision-aware Gen2 protocol when a generic Tag population $N>2$ is considered. Figure 6 shows the theoretical throughput per slot when varying the ratio of the number of slots per round $K$ over the Tag population $N$. Also in this case empirical values of $p_{C D}$ and $p_{C R}$ (0.83 and 0.9 respectively) are imposed for (3) and (4). The theoretical throughput gains achieved by Mode 1 and Mode 2 over Mode 0 when $K=N$ are $38 \%$ and $76 \%$, respectively.

\section{CONCLUSION}

We have considered a flexible testbed, made up of an opensource Software-Defined Radio (SDR) Reader and the programmable Intel WISP Tags, to implement techniques for multi-Tag acknowledgment under the assumption that collided Tag packets can be successfully recovered. The simple modifications to the EPC Gen2 protocol proposed in this paper experimentally demonstrate that performance of current RFID systems can be considerably enhanced when Tag collision recovery is performed at the Reader. Specifically, the average throughput per time slot is increased by $72 \%$ over the conventional Gen2 MAC scheme. Our attained results are in very good agreement with those provided via simulation in the literature, thus demonstrating the validity and effectiveness of the proposed approach. Among the advantages that such improved RFID system can bring in real-world applications, are the speed increase in conveyor belts and significant gains in the Reader power consumption. To the best of our knowledge, this is the first time that RFID performance with collision recovery is analyzed in real time with actual measurements.

\section{ACKNOWLEDGMENTS}

The authors would like to thank the Intel Labs Seattle for providing the WISP samples used in this work.

Author Vasileios Lakafosis would like to acknowledge the support of the Lilian Voudouri Foundation.

\section{REFERENCES}

[1] L. Simon, P. Saengudomlert, and U. Ketprom, "Speed adjustment algorithm for an RFID reader and conveyor belt system performing dynamic framed slotted aloha," 2008 IEEE International Conference on RFID, April 2008.
[2] J. Singh, E. Olsen, K. Vorst, and K. Tripp, "RFID tag readability issues with palletized loads of consumer goods," Packaging Technology and Science, 2009

[3] X. Lei, et al., "Efficient tag identification in mobile RFID systems," IEEE INFOCOM 2010, March 2010.

[4] EPCglobal, "EPC radio-frequency identify protocols - Class-1 Generation-2 UHF RFID protocol for communications at $860 \mathrm{MHz}-960$ $\mathrm{MHz}$, version 1.2.0, 2008

[5] L. G. Roberts, "Aloha packet system with and without slots and capture," SIGCOMM Computer Communications Review, 1975.

[6] A. F. Mindikoglu and A.-J. van der Veen, "Separation of overlapping RFID signals by antenna arrays," in Proceedings of the International Conference on Acoustics, Speech, and Signal Processing (ICASSP), pp. 2737-2740, 2008.

[7] B. Frey, "Source separation for uhf rfid," M.S Thesis, Delft and ETH, mentor Geert Leus, supervisor Helmut Bolskei, 2008

[8] J. Zhai and G.-N. Wang, "An anti-collision algorithm using twofunctioned estimation for RFID tags," in International Conference on Computational Science and Its Applications (ICCSA 2005), Springer Berlin / Heidelberg, pp. 702-711, 2005.

[9] P. V. Nikitin and K. V. S. Rao, "Theory and measurement of backscattering from RFID tags," IEEE Antennas and Propagation Magazine, vol. 48, no. 6, pp. 212-218, 2006.

[10] C. Angerer, R. Langwieser, and M. Rupp, "RFID reader receivers for physical layer collision recovery," IEEE Transactions on Communications, vol. 58, no. 12, pp. 3526-3537, Dec. 2010.

[11] J. Kimionis, A. Bletsas, A. G. Dimitriou, and G. N. Karystinos, "Inventory time reduction in Gen2 with single-antenna separation of FM0 RFID signals," in Proceedings of 2011 IEEE International Conference on RFID, April 2011.

[12] K. Fyhn, R. M. Jacobsen, P. Popovski, A. Scaglione, and T. Larsen, "Multipacket reception of passive UHF RFID tags: a communication theoretic approach," IEEE Transactions on Signal Processing, vol. 59, no. 9, pp. 4225-4237, 2011.

[13] R. S. Khasgiwale, R. U. Adyanthaya, and D. W. Engels, "Extracting information from tag collisions," in Proceedings of 2009 IEEE International Conference on RFID, April 2009.

[14] A. Sample et al., "Design of an RFID-based battery-free programmable sensing platform," IEEE Transactions on Instrumentation and Measurement, pp. 2608 -2615, 2008.

[15] M. Buettner and D. Wetherall, "A software radio-based UHF RFID reader for PHY/MAC experimentation," in Proceedings of 2011 IEEE International Conference on RFID, April 2011.

[16] Michael Buettner, Gen2 RFID Reader project page, Online: https://www.cgran.org/wiki/Gen2/.

[17] Ettus Research LLC, http://www.ettus.com/products/.

[18] GNU Radio, http://gnuradio.org/.

[19] L. Catarinucci, D. De Donno, M. Guadalaupi, F. Ricciato, and L. Tarricone, "Performance analysis of passive UHF RFID tags with GNU Radio," IEEE International Symposium on Antennas and Propagation (APSURSI), pp. 541-544, Spokane, WA, USA, July 2011.

[20] D. De Donno, F. Ricciato, L. Catarinucci, A. Coluccia and L. Tarricone, "Challenge: towards distributed RFID sensing with software-defined radio," Sixteenth annual International Conference on Mobile Computing and Networking (MobiCom '10), pp. 97-104, Chicago, Illinois, USA, September 2010

[21] D. De Donno, F. Ricciato, L. Catarinucci, and L. Tarricone, "Design and applications of a software-defined listener for UHF RFID systems," IEEE MTT-S International Microwave Symposium Digest, Baltimore, MD, USA, June 2011. 